\title{
SOIL MECHANICS AND PLASTIC ANALYSIS OR LIMIT DESIGN*
}

\author{
BY \\ D. C. DRUCKER AND W. PRAGER \\ Brown University
}

1. Introduction. Problems of soil mechanics involving stability of slopes, bearing capacity of foundation slabs and pressures on retaining walls are often treated as problems of plasticity. The soil is replaced by an idealized material which behaves elastically up to some state of stress at which slip or yielding occurs. The shear stress required for simple slip is often considered to depend upon the cohesion and linearly upon the normal pressure on the slip surface. In more complete plane investigations an extended Coulomb's rule is used,** (Fig. 1)

$$
R=c \cos \varphi-\frac{\sigma_{x}+\sigma_{y}}{2} \sin \varphi,
$$

where $R$ is the radius of Mohr's circle at slip, the maximum shearing stress $\left[\left(\sigma_{x}-\sigma_{y}\right)^{2} / 4+\right.$ $\left.\tau_{x y}^{2}\right]^{1 / 2} ; c$ is the cohesion; $c \cos \varphi$ is the radius of Mohr's circle at slip when the mean normal stress, $\left(\sigma_{x}+\sigma_{y}\right) / 2$, in the plane is zero, and $\varphi$ is the angle between the tangent to the Mohr's circles at slip and the negative $\sigma$ axis.

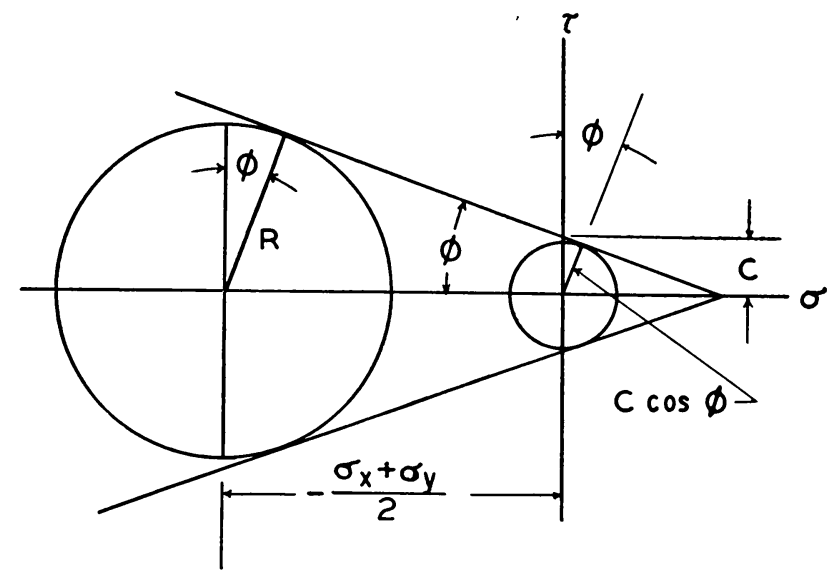

FIG. 1. Mohr-Coulomb hypothesis.

The purpose of this note is to discuss the implications of assuming the soil to be a perfectly plastic body. The validity of this assumption is not at issue. No account is taken of such important practical matters as the effect of water in the soil or of the essentially different behavior of various constituents such as clay and sand. All that is sought is a theory consistent with the basic assumption. Comparison of the predictions of such a theory with the actual behavior of soil will give an indication of the value of the idealization.

*Received Nov. 19, 1951. The results presented in this paper were obtained in the course of research sponsored by the Office of Naval Research under Contract N7onr-35801 with Brown University.

${ }^{* *}$ K. Terzaghi, Theoretical soil mechanics, John Wiley and Sons, 1943, pp. 22, 59. 
2. Yield function and stress-strain relation. A yield function which is a proper generalization of the Mohr-Coulomb hypothesis (1) is

$$
f=\alpha J_{1}+J_{2}^{1 / 2}=k,
$$

where $\alpha$ and $k$ are positive constants at each point of the material; $J_{1}$ is the sum of the principal stresses:

$$
J_{1}=\sigma_{1}+\sigma_{2}+\sigma_{3}=\sigma_{x}+\sigma_{\nu}+\sigma_{z} \equiv \sigma_{11}+\sigma_{22}+\sigma_{33} \equiv \sigma_{i i} ;
$$

$J_{2}$ is the second invariant of the stress deviation:

$$
J_{2}=\frac{1}{2} s_{i j} s_{i j}=\frac{1}{6}\left[\left(\sigma_{x}-\sigma_{y}\right)^{2}+\left(\sigma_{y}-\sigma_{z}\right)^{2}+\left(\sigma_{z}-\sigma_{x}\right)^{2}\right]+\tau_{x y}^{2}+\tau_{y z}^{2}+\tau_{z x}^{2} .
$$

The stress deviation is defined as $s_{i j}=\sigma_{i j}-\left(J_{1} / 3\right) \delta_{i j}$, where $\delta_{i j}$ is the Kronecker delta, zero for $i \neq j$ and unity for $i=j$. For example, $s_{11} \equiv s_{x}=\frac{2}{3}\left[\sigma_{x}-\frac{1}{2}\left(\sigma_{\nu}+\sigma_{z}\right)\right], s_{12} \equiv$ $s_{x y}=\tau_{x y}$.

As is well known, the yield surface, $f=k$, in principal stress space is a right circular cylinder for the Mises criterion $(\alpha=0)$. For $\alpha>0$, the surface is a right circular cone with its axis equally inclined to the coordinate axes and with its apex in the tension octant. A generalization of (1) as a modified Tresca or maximum shear stress criterion is also permissible, but will not be discussed here. It leads to a pyramid instead of a cone.

According to the concept of plastic potential, ${ }^{*}$ the stress-strain relation corresponding to the yield function (2) is

$$
\epsilon_{i j}^{\prime p}=\lambda \partial f / \partial \sigma_{i j}
$$

where $\epsilon_{i j}^{\prime p}$ is the plastic strain rate and $\lambda$ is a positive factor of proportionality which may assume different values for different particles. Substituting the expression (2) for $f$, we obtain

$$
\epsilon_{i j}^{\prime p}=\lambda\left[\alpha \delta_{i j}+s_{i j} / 2 J_{2}^{1 / 2}\right] .
$$

A very important feature of Eq. (4) is that the plastic rate of cubical dilatation is

$$
\epsilon_{i i}^{\prime p}=3 \alpha \lambda \text {. }
$$

Equation (5) shows that plastic deformation must be accompanied by an increase in volume if $\alpha \neq 0$. This property is known as dilatancy.**

3. Collapse or limit design theorems. It has been shown previously*** that when the limit load is reached for any body or assemblage of bodies of perfectly plastic material, collapse takes place at constant stress. This means that at the instant of collapse the strain rates are purely plastic. The assumption is made, just as in most problems of elasticity, that changes in geometry are negligible.

As we shall deal only with collapse or limiting states, $\epsilon_{i j}^{\prime p}$ equals $\epsilon_{i j}^{\prime}$, the total strain rate.

The major limit theorems for stress boundary value problems are:

(i) collapse will not occur if any state of stress can be found which satisfies the

${ }^{*}$ R. v. Mises, Mechanik der plastischen Formaenderung von Kristallen, Z. angew. Math. Mech. 8, 161-185 (1928).

** M. Reiner, A mathematical theory of dilatancy, Am. Journ. of Math. 67, 350-362 (1945).

***D. C. Drucker, H. J. Greenberg, W. Prager, Extended limit design theorems for continuous media Q. Appl. Math. 9, 381-389 (1952). 
equations of equilibrium and the boundary conditions on stress and for which $f<k$ everywhere; and

(ii) collapse must occur if for any compatible flow pattern, considered as plastic only, the rate at which the external forces do work on the body equals or exceeds the rate of internal dissipation.

The theorems are valid when frictional surface tractions are present if there is no slip or if the frictional forces are known.

Discontinuous stress or flow states are permissible and are generally convenient for computational purposes.

4. Plane strain. The yield function (2) will first be shown to reduce to the MohrCoulomb rule in the case of plane strain. In this case $\epsilon_{33}^{\prime}, \epsilon_{13}^{\prime}, \epsilon_{23}^{\prime}$ (or $\epsilon_{z}^{\prime}, \gamma_{x z}^{\prime}, \gamma_{y z}^{\prime}$ in engineering notation) vanish. Therefore, from (4), $s_{13}$ and $s_{23}\left(\tau_{x z}, \tau_{y z}\right)$ are zero, and

Thus,

$$
s_{33}=-2 \alpha J_{2}^{1 / 2} .
$$

and

$$
J_{1}=\frac{3}{2}\left(\sigma_{11}+\sigma_{22}\right)-3 \alpha J_{2}^{1 / 2},
$$

$$
J_{2}=\left[\left(\frac{\sigma_{x}-\sigma_{y}}{2}\right)^{2}+\tau_{x y}^{2}\right] /\left(1-3 \alpha^{2}\right) .
$$

Substituting (7) and (8) into the yield function (2), we find

$$
\begin{gathered}
f=3 \alpha \frac{\sigma_{x}+\sigma_{y}}{2}+\left(1-3 \alpha^{2}\right) J_{2}^{1 / 2}=k \\
\frac{k}{\left(1-3 \alpha^{2}\right)^{1 / 2}}=\frac{3 \alpha}{\left(1-3 \alpha^{2}\right)^{1 / 2}} \frac{\sigma_{x}+\sigma_{y}}{2}+\left[\left(\frac{\sigma_{x}-\sigma_{y}}{2}\right)^{2}+\tau_{x y}^{2}\right]^{1 / 2} .
\end{gathered}
$$

Equation (9) becomes identical with Eq. (1) if we set

and hence

$$
c=\frac{k}{\left(1-12 \alpha^{2}\right)^{1 / 2}}, \quad \frac{3 \alpha}{\left(1-3 \alpha^{2}\right)^{\overline{1 / 2}}}=\sin \varphi
$$

$$
\frac{\left(1-12 \alpha^{2}\right)^{1 / 2}}{\left(1-3 \alpha^{2}\right)^{1 / 2}}=\cos \varphi
$$

A clearer picture of the meaning of the plasticity relations is obtained by considering a plane velocity pattern as shown in Fig. 2. The upper portion is supposed to translate

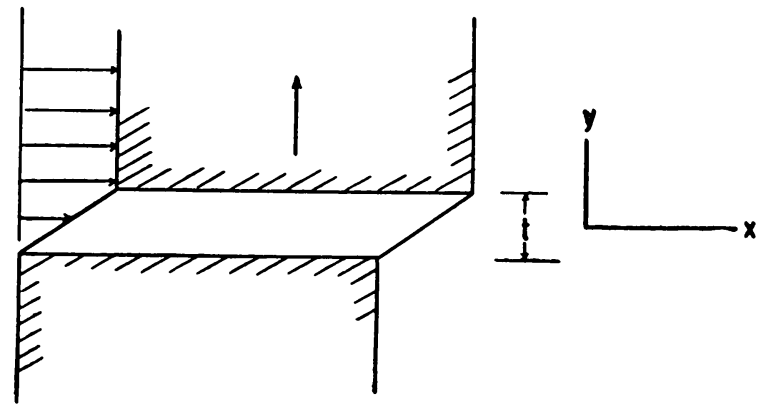

Fig. 2. "Simple" slip. 
as a rigid body while the lower part is fixed. If the transition layer between is made thin enough, Fig. 2 represents simple slip. The essential feature is that $\epsilon_{x}^{\prime}=0$. As $\epsilon_{z}^{\prime}$ is zero, $\epsilon_{\nu}^{\prime}$ cannot be zero. In fact, from Eq. (5), $\epsilon_{\nu}^{\prime}=3 \alpha \lambda$ and the upper shaded region not only moves to the right but it also must move upward. Such volume expansion accompanying slip is a well known property of reasonably packed granular material. It is an interesting and in fact a necessary result of plasticity theory that if the yield point in shear depends upon the mean normal stress, slip is accompanied by volume change. This necessary relation is an essential difference between a plastic material and an assemblage of two solid bodies with a sliding friction contact.*

Figure 1 shows clearly that the shearing stress on the slip surface is not the maximum shearing stress $R$ but is $R \cos \varphi$.

It is interesting to observe that $\alpha$ is not completely free because $12 \alpha^{2}$ cannot exceed unity. As shown by Eqs. (10) this is equivalent to restricting $\sin \varphi$ to be no more than unity.

5. Rate of dissipation of energy. The rate of dissipation of mechanical energy per unit volume is

$$
D=\sigma_{i i} \epsilon_{i j}^{\prime p}=\sigma_{i i} \lambda \frac{\partial f}{\partial \sigma_{i j}}=\lambda f=\lambda k .
$$

As the dissipation is to be computed from a plastic strain rate pattern, it is desirable to express $\lambda$ in terms of strain rate. It follows from (3) that

$$
\boldsymbol{\epsilon}_{i j}^{\prime} \epsilon_{i j}^{\prime}=\lambda^{2} \frac{\partial f}{\partial \sigma_{i j}} \frac{\partial f}{\partial \sigma_{i j}}=\lambda^{2}\left(3 \alpha^{2}+\frac{1}{2}\right)
$$

Substituting in (11)

$$
D=\frac{k\left(\epsilon_{i i}^{\prime} \epsilon_{i j}^{\prime}\right)^{1 / 2}}{\left(3 \alpha^{2}+1 / 2\right)^{1 / 2}}=\frac{c \cos \varphi}{\left(1+\sin ^{2} \varphi\right)^{1 / 2}}\left[2 \epsilon_{x}^{\prime 2}+2 \epsilon_{y}^{\prime 2}+2 \epsilon_{z}^{\prime 2}+\gamma_{x y}^{\prime 2}+\gamma_{y z}^{\prime 2}+\gamma_{z x}^{\prime 2}\right]^{1 / 2}
$$

which for the plane strain case may be written as

$$
D=c \cos \varphi\left[\left(\epsilon_{x}^{\prime}-\epsilon_{y}^{\prime}\right)^{2}+{\gamma_{x y}^{\prime 2}}^{1 / 2}\right.
$$

For purposes of calculation it is convenient to consider a transition layer as in Fig. 2 to be a simple discontinuity. However, for $\alpha \neq 0$ a discontinuity $\delta u^{\prime}$ in tangential velocity must be accompanied by a separation or discontinuity $\delta v^{\prime}$ in normal velocity. A transition layer of appreciable thickness therefore must be present in a soil while there is no need for such a layer in a Prandtl-Reuss material where $\alpha=0$.

The rate of dissipation of work per unit area of discontinuity surface is

$$
D_{A}=\tau \delta u^{\prime}+\sigma \delta v^{\prime}
$$

where $\tau$ is the shearing stress and $\sigma$ the normal stress (tension is positive) on the surface. The same result can be obtained by taking the limit of $t D$, Eqs. $(13,14)$ as $t$, the thickness of the transition layer goes to zero while $t \epsilon_{12}^{\prime} \equiv t \frac{1}{2} \gamma_{x y}^{\prime}=\frac{1}{2} \delta u^{\prime}$ and $t \epsilon_{22}^{\prime}=t \epsilon_{\nu}^{\prime}=\delta v^{\prime}$. It follows that

$$
\delta v^{\prime}=\frac{\epsilon_{22}^{\prime}}{2 \epsilon_{12}^{\prime}} \delta u^{\prime}=\frac{3 \alpha}{\left(1-12 \alpha^{2}\right)^{1 / 2}} \delta u^{\prime} \equiv \delta u^{\prime} \tan \varphi .
$$

${ }^{*}$ D. C. Drucker, Some implications of work hardening and ideal plasticity, Q. Appl. Math. 7, 411-418 (1950). 
Also

$$
D_{A}=\frac{k \delta u^{\prime}}{\left(1-12 \alpha^{2}\right)^{1 / 2}}=c \delta u^{\prime} .
$$

These results apply equally well to a curved surface of discontinuity because there is no essential distinction locally as the thickness of the transition layer approaches zero.

6. Dilatation. The rate of cubical dilatation, $\epsilon_{i i}^{\prime}$, which accompanies plastic deformation is given by Eq. (5). Substitution of (12) leads to

$$
\begin{aligned}
\epsilon_{i i}^{\prime} & =\frac{3 \alpha}{\left(1+6 \alpha^{2}\right)^{1 / 2}}\left(2 \epsilon_{i j}^{\prime} \epsilon_{i j}^{\prime}\right)^{1 / 2} \\
& =\frac{\sin \varphi}{\left(1+\sin ^{2} \varphi\right)^{1 / 2}}\left(2 \epsilon_{i j}^{\prime} \epsilon_{i j}^{\prime}\right)^{1 / 2} .
\end{aligned}
$$

For plane strain, we obtain the rate of dilatation

$$
\Delta^{\prime}=\epsilon_{x}^{\prime}+\epsilon_{y}^{\prime}=\frac{\sin \varphi}{\left(1+\sin ^{2} \varphi\right)^{1 / 2}}\left(2 \epsilon_{x}^{\prime 2}+2 \epsilon_{y}^{\prime 2}+{\gamma_{x y}^{\prime 2}}^{\prime 2}\right)^{1 / 2}
$$

or

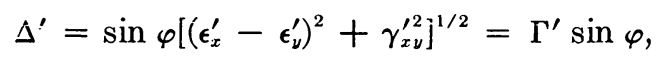

where $\Gamma^{\prime}$ is the maximum shear rate.

In terms of principal strain rates for plane strain, $\epsilon_{1}^{\prime}, \epsilon_{2}^{\prime}$, where $\epsilon_{1}^{\prime}>\epsilon_{2}^{\prime}$

$$
\epsilon_{1}^{\prime}=\epsilon_{2}^{\prime} \frac{1+\sin \varphi}{1-\sin \varphi}=\epsilon_{2}^{\prime} \tan ^{2}\left(45+\frac{\varphi}{2}\right) .
$$

7. Simple discontinuous solutions. Any soil mass, in particular the slope shown in Fig. 3, may fail by rigid body "sliding" motion in two simple ways. As is well known, the surfaces of discontinuity, idealizations of discontinuity layers, are planes and circular cylinders for a Prandtl-Reuss material, $\alpha=0$. When $\alpha \neq 0$, a plane discontinuity surface is still permissible but the circle is replaced by a logarithmic spiral which is at an

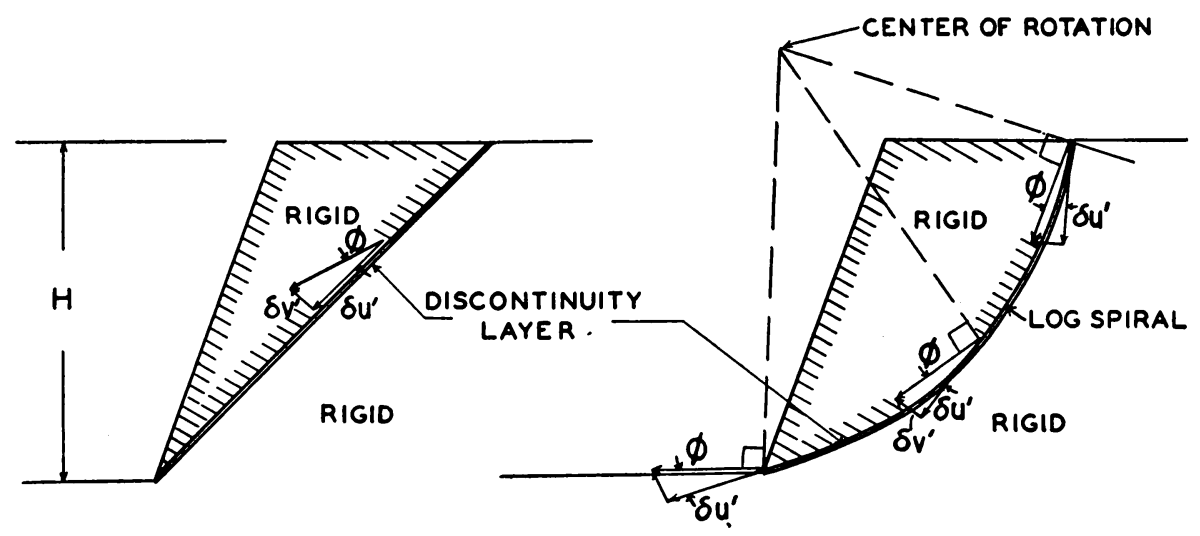

FIG. 3. Rigid body "slide" motions.

angle $\varphi$ to the radius from the center of rotation. The circle is not a permissible surface for rigid body "sliding" because a discontinuity in tangential velocity requires a separa- 
tion velocity. For a "slide" discontinuity the angle between the velocity vector and the discontinuity surface is $\varphi$, as is seen from Eq. (16).

8. Example: Critical height of vertical bank. The computation of the critical height $H_{c}$ of a vertical bank, Fig. 4, will serve as an illustration of the procedure consistent with plasticity theory. No attempt will be made here to get an exact plasticity solution to this problem for even if it could be obtained it would not be helpful for more complicated slopes. Instead, the upper and lower bound techniques of limit design will be used.

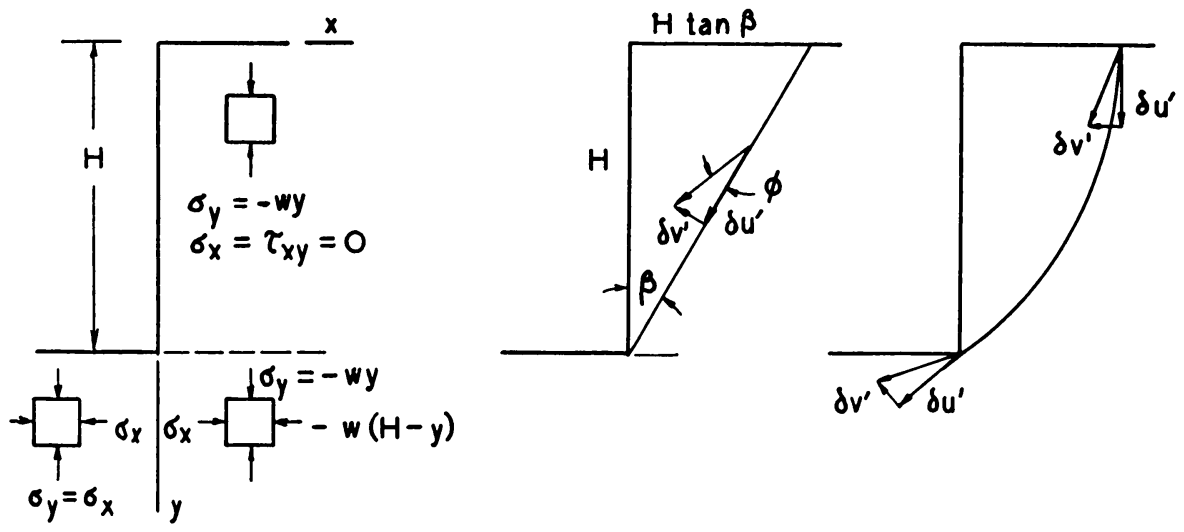

FIG. 4. (a) An equilibrium solution, (b) Plane, (c) Log. spiral compatible solutions.

Denoting the specific weight of the soil by $w$, Fig. 4 a shows a discontinuous equilibrium solution in which the maximum shearing stress is $w H / 2$ at the lower ground level, where the average normal stress is $-w H / 2$. It should be remembered that the stress field need bear no resemblance to the actual state of stress. Equilibrium alone must be satisfied in addition to the yield condition (1) or (2) for $H$ to be safe. From Eq. (1)

$$
\frac{w H}{2}=c \cos \varphi+\frac{w H}{2} \sin \varphi
$$

or a lower bound on the critical height $H_{c}$ is given by

$$
H_{c} \geq H=\frac{2 c}{w} \frac{\cos \varphi}{1-\sin \varphi}=\frac{2 c}{w} \tan \left(45+\frac{\varphi}{2}\right)
$$

An upper bound on $H_{c}$ may be found by taking a plane slide as a velocity pattern, Fig. 4b. Equating external rate of work to the dissipation Eq. (18) gives

so that

$$
\frac{w H^{2} \tan \beta}{2} \frac{\delta u^{\prime}}{\cos \varphi} \cos (\varphi+\beta)=\frac{c H}{\cos \beta} \delta u^{\prime},
$$

$$
H_{c} \leq H=\frac{2 c}{w} \frac{\cos \varphi}{\sin \beta \cos (\varphi+\beta)} .
$$

minimizing the right hand side gives

$$
\beta=45-\frac{\varphi}{2}
$$


and

$$
H_{c} \leq \frac{4 c}{w} \tan \left(45+\frac{\varphi}{2}\right)
$$

The usual theory furnishes the right-hand side of (25) as the critical height. However there is a factor of 2 between the upper bound (25) and the lower bound (22) for $H_{c}$.

The upper bound can be improved by considering a rotational discontinuity (logarithmic spiral, Fig. 4c) instead of the translational type.

9. A modified stress criterion. The large discrepancy between the lower and upper bounds derived from reasonable stress and velocity fields (Fig. 4a and Figs. 4b, c) has a simple explanation. An improvement in the static or equilibrium answer would require adding stresses which will result in tensile stress in the soil. Nothing in the MohrCoulomb criterion, Fig. 1 rules out a moderate amount of tension. If it is felt that soil cannot take any tension, then the yield criterion must be altered to take into account explicitly that

$$
-\frac{\sigma_{x}+\sigma_{u}}{2} \geq\left[\left(\frac{\sigma_{x}}{2}-\sigma_{\nu}\right)^{2}+\tau_{x y}^{2}\right]^{1 / 2}=R
$$

As before, if rigid body discontinuous solutions are considered, one strain rate, say $\epsilon_{11}^{\prime}$, is zero. The relation between the normal and the shearing stress on a surface of discontinuity is now given by Fig. 5 instead of Fig. 1. The yield function and the re-

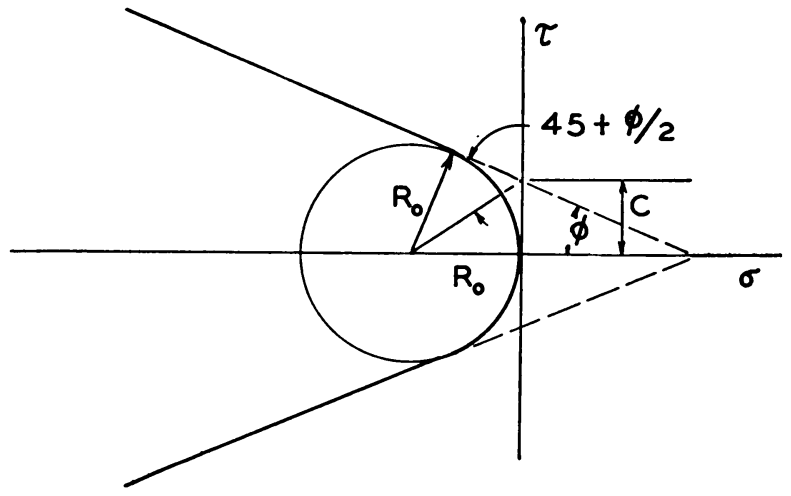

FIG. 5. Modified criterion for stress on discontinuity surface $R_{0}=c \tan (45+\phi / 2)$

sulting velocity relations previously derived apply only for the straight line portion of the limiting curve.

In general, the rate of dissipation, $D_{A}$, given by Eq. (15), may be interpreted as the dot product of a stress vector having rectangular Cartesian components $\sigma, \tau$ with a velocity vector having components $\delta v^{\prime}, \delta u^{\prime}$. The stress vector is shown on Fig. 6 as $O P$ or $O Q$ and the velocity vector is normal to the limiting curve. The normality condition holds because $\sigma$ and $\tau$ are the only stress components which do work. ${ }^{*}$ Considering the stress vector $O Q$ to be the sum of $O O^{\prime}$ and $O^{\prime} Q$, we have

$$
D_{A}=R_{0}\left\{\left[\left(\delta u^{\prime}\right)^{2}+\left(\delta v^{\prime}\right)^{2}\right]^{1 / 2}-\delta v^{\prime}\right\}=c \delta u^{\prime} \frac{\tan (45+\varphi / 2)}{\tan (45+\theta / 2)},
$$

${ }^{*}$ D. C. Drucker, A more fundamental approach to plastic stress-strain relations, to appear in Proceedings, 1st U. S. National Congress for Applied Mechanics. 


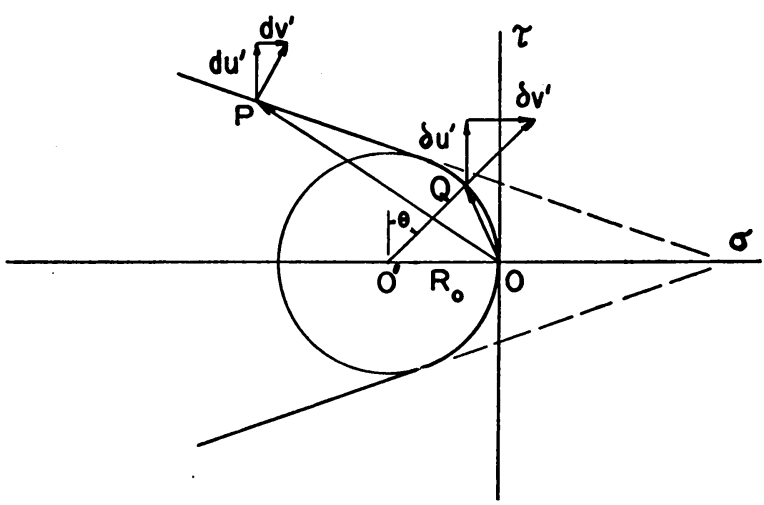

FIG. 6. Rate of dissipation on discontinuity surface is $\sigma \delta v^{\prime}+\tau \delta u$

where

$$
\begin{gathered}
\tan \theta=\frac{\delta v^{\prime}}{\delta u^{\prime}} \geq \tan \varphi \\
R_{0}=c \tan (45+\varphi / 2)
\end{gathered}
$$

If Fig. $4 \mathrm{~b}$ is modified by taking the angle between the velocity vector and the discontinuity line as $\theta$ instead of $\varphi$, the results are not altered. However, if a very crude picture is employed of rotation about a point below the lower ground level, Fig. 7, the upper bound is easily reduced to

$$
H_{c} \leq H=\frac{2.5}{w} c \tan \left(45+\frac{\varphi}{2}\right) .
$$

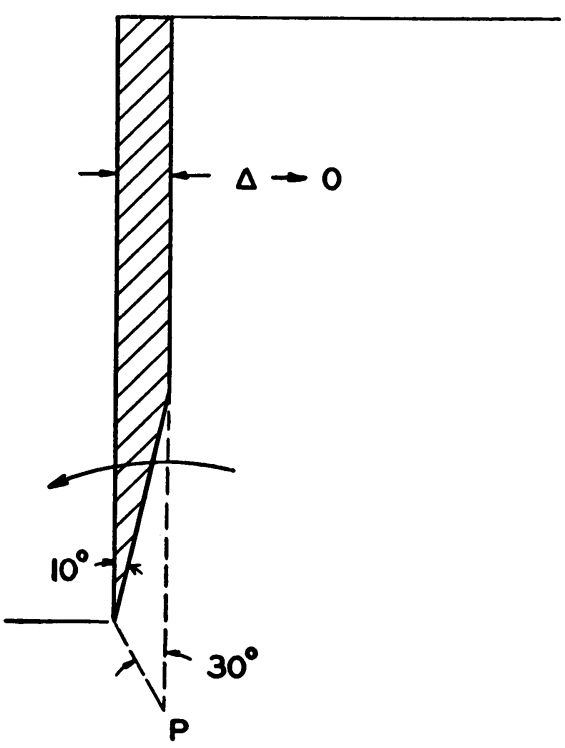

Fig. 7. Rotation about $P$, soil of zero tensile strength. 
Further reduction is possible with other discontinuous patterns but the concept of a soil unable to take tension clearly requires much additional study.

10. Conclusion. It has been shown that the implications of plasticity theory and limit design for soil mechanics are indeed far reaching. Circular sliding surfaces are replaced by logarithmic spirals in a material whose yield stress in shear depends upon normal stress. Volume expansion is seen to be a necessary accompaniment to shearing deformation. Low critical heights are found for slopes when the soil is assumed to be unable to take tension. 